PoS $\quad \begin{aligned} & \text { PROCEEDINGS } \\ & \text { OF SCIENCE }\end{aligned}$

\title{
Wilson twisted mass fermions in the epsilon regime
}

\section{K. Jansen, A. Nube,} DESY Zeuthen, Platanenallee 6 D-15738 Zeuthen, Germany

E-mail: karl.jansen@desy.de,annube@ifh.de

\section{A. Shindler*}

Theoretical Physics Division,

Dept. of Mathematical Sciences,

University of Liverpool

Liverpool L69 7ZL, UK

E-mail: shindler@liv.ac.uk

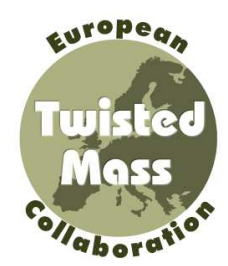

In this proceeding contribution we report on the ongoing effort to understand and simulate Wilson twisted mass fermions in the so called $\varepsilon$ regime.

The XXVI International Symposium on Lattice Field Theory

July 14 - 19, 2008

Williamsburg, Virginia, USA

${ }^{*}$ Speaker. 


\section{Introduction}

Simulations in the $\varepsilon$ regime are complementary to standard large volume simulations. They allow to extract low energy constants of the chiral Lagrangian, in some cases with less contaminations from chiral logs coming from higher order corrections. For a long time it has been thought that simulations in the $\varepsilon$ regime are restricted to chirally invariant lattice formulations. In ref. [1] we have argued that actually this is not the case, and that simulations in the $\varepsilon$ regime can be performed also with not chiral invariant lattice actions, as Wilson-like fermions.

In particular in [1] we suggested that a combination of algorithmic and theoretical understanding of Wilson twisted mass makes possible to think and actually perform simulations in the $\varepsilon$ regime with Wilson twisted mass fermions.

Recently it has been shown that with suitable and related algorithmic ideas [2] it possible to reach or get close to the $\varepsilon$ regime also with improved Wilson fermions. At this lattice conference further results in this directions have been presented [3].

In this proceeding we extend to a second lattice spacing and to NLO the analysis performed in [1]. Our setup is a $L^{3} \times T$ Euclidean lattice with spacing $a$. The lattice action

$$
S[\chi, \bar{\chi}, U]=S_{G}[U]+S_{F}[\chi, \bar{\chi}, U],
$$

is a combination the so called tree-level improved Symanzik gauge action [4]

$$
S_{G}[U]=\frac{\beta}{3} \sum_{x}\left\{b_{0} \sum_{\mu<v} \mathbb{R e} \operatorname{Tr}\left[\mathbb{1}-P^{(1 \times 1)}(x ; \mu, v)\right]+b_{1} \sum_{\mu \neq v} \mathbb{R e} \operatorname{Tr}\left[\mathbb{1}-P^{(2 \times 1)}(x ; \mu, v)\right]\right\},
$$

with

$$
b_{0}=1-8 b_{1}, \quad b_{1}=-\frac{1}{12}
$$

and Wilson twisted mass [5]

$$
S_{\mathrm{F}}[\chi, \bar{\chi}, U]=a^{4} \sum_{x} \bar{\chi}(x)\left[D_{\mathrm{W}}+i \mu_{\mathrm{q}} \gamma_{5} \tau^{3}\right] \chi(x),
$$

where

$$
D_{\mathrm{W}}=\frac{1}{2}\left\{\gamma_{\mu}\left(\nabla_{\mu}+\nabla_{\mu}^{*}\right)-a \nabla_{\mu}^{*} \nabla_{\mu}\right\}+m_{0}
$$

The basic idea of [1] is that sampling all topological sectors in the ensemble generation, it is not needed to have an unambiguous definition of topology at finite lattice spacing. To achieve this goal it was suggested [1] to use a PHMC algorithm [6] treating the low modes exactly and reweighting the observables. This could allow to perform simulations at very low quark masses without encountering instabilities or metastabilities.

\section{2. $\varepsilon$ expansion}

Lowering the quark mass at finite lattice spacing with Wilson-like fermions requires a detailed understanding of the interplay between genuine chiral behaviour induced by the 'pion' dynamics and the one generated by cutoff effects. A review on the phase diagram and cutoff effects with Wilson twisted mass (Wtm) can be found in ref. [7]. In the $\varepsilon$ regime this is equivalent to say that 
it is necessary to understand the coupling of the zero modes with the relevant operators describing the effect of the lattice artifacts. The actual values of the lattice spacing, the physical volume and the quark mass determine the appropriate power counting that ought to be used to perform computations using chiral effective theories. In the continuum the exact integration over the constant zero modes can be achieved in the chiral effective theory modifying the $p$ regime power counting, resulting in the so called $\varepsilon$ expansion where the would-be pion mass is small compared to the linear size of the box

$$
\frac{1}{T}=\mathrm{O}(\varepsilon), \quad \frac{1}{L}=\mathrm{O}(\varepsilon), \quad M_{\pi}=\mathrm{O}\left(\varepsilon^{2}\right) .
$$

As a result of the exact integration the order parameter, or the equivalent ratio $R=\frac{\langle\bar{q} q\rangle}{B_{0} F^{2}}$ vanishes in the chiral limit at fixed finite volume [8], obtaining restoration of chiral symmetry. One possible way to include the effects of the lattice artifacts in this analysis is to include with an appropriate power counting the lattice spacing. Here we modify the standard power counting in the following way [9]

$$
M=O\left(\varepsilon^{4}\right), \quad \frac{1}{L}=O(\varepsilon), \quad \frac{1}{T}=O(\varepsilon) \quad a^{2}=O\left(\varepsilon^{4}\right),
$$

where $M$ indicates generically a quark mass. The partition function at leading order reads

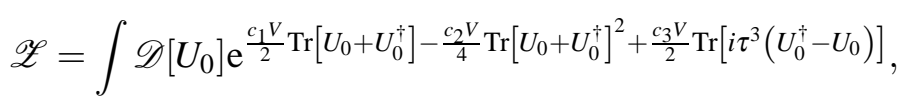

where the scaling variables are

$$
z_{1}=c_{1} V=B_{0} F^{2} m^{\prime} V, \quad z_{2}=c_{2} V=-\frac{F^{2} w^{\prime} V a^{2}}{4}, \quad z_{3}=c_{3} V=B_{0} F^{2} \mu_{\mathrm{R}} V .
$$

To argue that this a proper power counting for actual numerical simulations we list here some values

$$
\begin{aligned}
& M \simeq 5 \mathrm{MeV}, \quad a \simeq 0.1 \mathrm{fm}, \quad L \simeq 1.5 \mathrm{fm} \\
& F \simeq 90 \mathrm{MeV}, \quad B_{0} \simeq 5.5 \mathrm{GeV}, \quad\left|w^{\prime}\right| \simeq(570 \mathrm{MeV})^{4} .
\end{aligned}
$$

Using these values to estimate the relevant scaling variables in this regime one obtains

$$
M F^{2} B_{0} V \simeq 0.75, \quad a^{2} F^{2}\left|w^{\prime}\right| V \simeq 0.75, \quad \frac{M B_{0}}{a^{2}\left|w^{\prime}\right|} \simeq 1,
$$

which seems to indicate that this is an appropriate power counting. ${ }^{1}$ The chiral condensate can be computed in the standard way

$$
R=\frac{1}{N_{\mathrm{f}}} \frac{\partial}{\partial z_{3}} \log \mathscr{Z}, \quad z_{1}=0,
$$

and fig. 1 shows the quark mass (left plot) and lattice spacing (right plot) dependence of the chiral condensate. We can certainly conclude that the dependence on the quark mass is, as expected, smooth, and the cutoff effects are under control. Extension of this computation to NLO including standard two-point functions is currently in progress [9]. The power counting introduced is general and valid also for plain Wilson fermions $\left(z_{3}=0\right)$. The same power counting could be used to develop an expansion with staggered fermions and to check the chiral properties of the spectrum in the presence of roots of the staggered determinant.

\footnotetext{
${ }^{1}$ If the lattice spacing is much smaller a different power counting ought to be used where the lattice artifacts only appear at NNLO.
} 

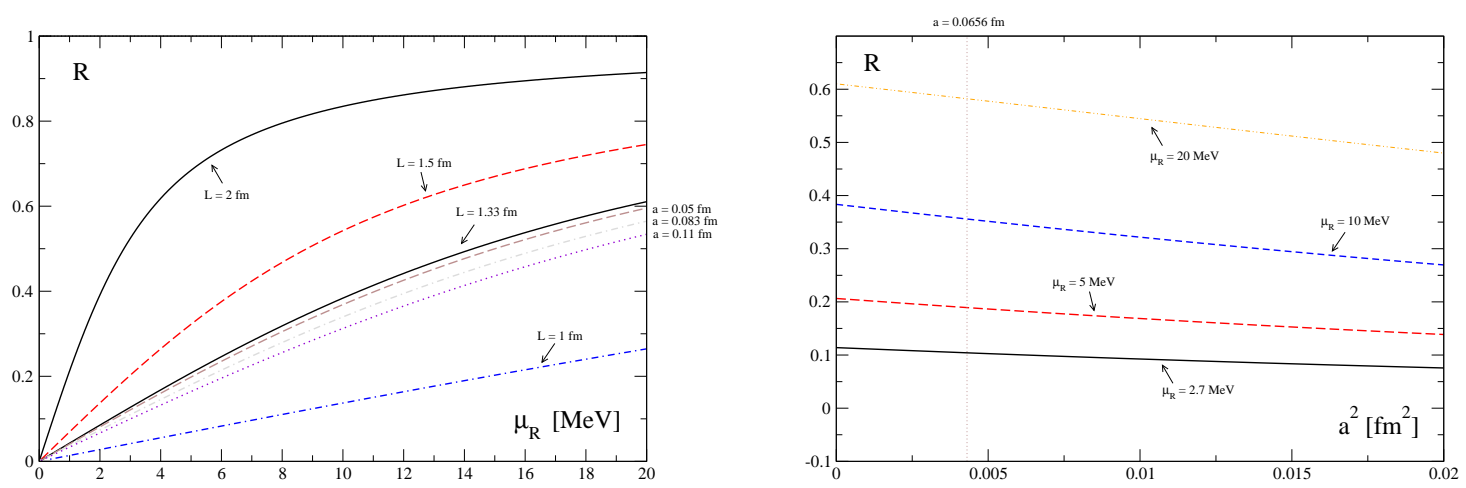

Figure 1: Quark mass (left plot) and lattice spacing (right plot) dependence for the single flavour chiral condensate normalized with its LO value in the continuum and infinite volume.

\begin{tabular}{|l|c|l|l|c|}
\hline$\beta$ & $\kappa$ & $L / a$ & $T / a$ & $a \mu_{\mathrm{q}}$ \\
\hline 4.05 & 0.157010 & 20 & 40 & 0.00039 \\
\hline
\end{tabular}

\begin{tabular}{|l|l|l|l|}
\hline$N_{\text {traj }}$ & $N_{\text {ana }}$ & $\tau_{\text {int }}(P)$ & $\tau_{\text {int }}\left(m_{\text {PCAC }}\right)$ \\
\hline 2500 & 421 & $\sim 0.5$ & $\sim 0.5$ \\
\hline
\end{tabular}

\begin{tabular}{|l|c|l|c|}
\hline$r_{0} / a$ & $a[\mathrm{fm}]$ & $L[\mathrm{fm}]$ & $a m_{\mathrm{PCAC}}$ \\
\hline $6.61(3)$ & $0.0656(11)$ & 1.31 & $0.00045(12)$ \\
\hline
\end{tabular}

Table 1: Summary of the simulation setup and of the basic ensemble parameters.

\section{Numerical results}

Details on the algorithm used to generate the gauge ensemble can be found in ref. [1]. In this proceeding we extend the results obtained in [1] to a second lattice spacing with a NLO analysis. The inversions for the quark propagator have been performed with a stochastic $Z_{2} \times Z_{2}$ source located randomly along euclidean time. Table 3 summarizes the simulation setup. In the left plot of fig. 2 we show in the first strip the plaquette MC history and its distribution. In the second strip we show the MC history and distribution of the lowest eigenvalue, compared with the value of the infrared cutoff (horizontal red line) provided by the twisted mass. In the third strip we show the MC history of the reweighting factor and its distribution. One crucial parameter for stability issues and for controlling discretization errors is the PCAC mass. In the right plot of fig. 2 we show the MC history and the distribution of the PCAC mass at $x_{0}=T / 4$, together with the euclidean time dependence of the PCAC mass. It is remarkable the almost absence of boundary $\mathrm{O}(a)$ cutoff effects. The analysis gives with the corresponding $\mathrm{Z}$ factors [10]

$$
a m_{\mathrm{PCAC}}=0.00045(12) \quad \Rightarrow \quad a M_{\mathrm{R}}^{\overline{\mathrm{MS}}}(2 \mathrm{GeV})=0.0012(2),
$$

where

$$
M_{\mathrm{R}}^{\overline{\mathrm{MS}}}(2 \mathrm{GeV})=\frac{1}{Z_{\mathrm{P}}} M \quad M=\sqrt{\left(Z_{\mathrm{A}} m_{\mathrm{PCAC}}\right)^{2}+\mu_{\mathrm{q}}^{2}} .
$$

We are clearly not at full twist. It is important to remark that this is not so relevant in the regime where chiral symmetry is restored. Automatic $\mathrm{O}(a)$ improvement [11] actually holds in a finite 

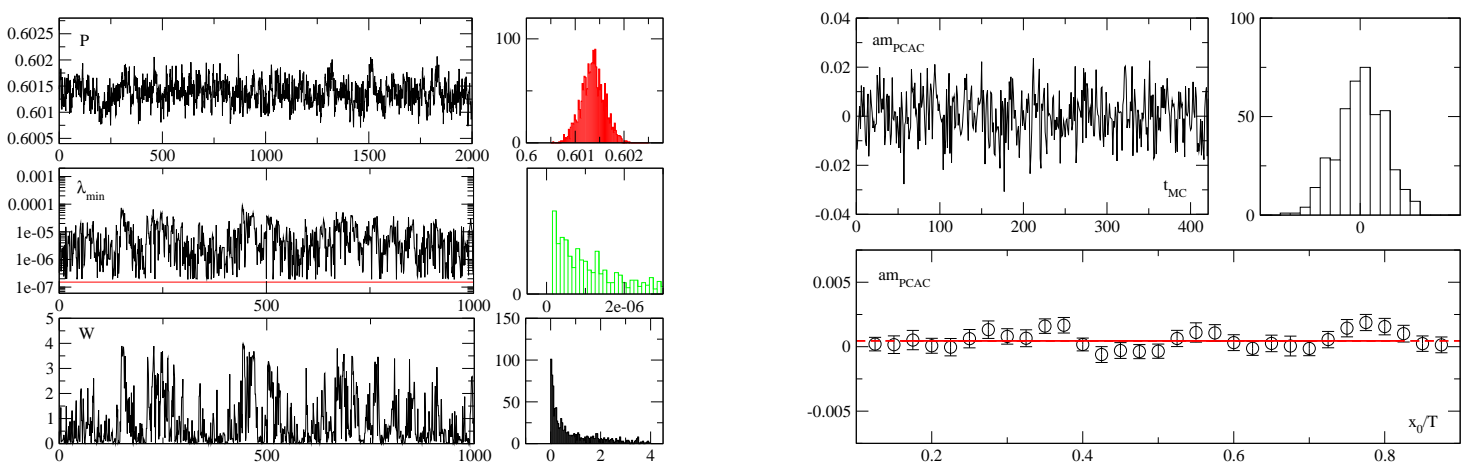

Figure 2: Left plot: MC histories and distributions of the plaquette (first strip), smallest eigenvalue (second strip) and reweighting factor (third strip). The smallest eigenvalue is compared with the infrared cutoff provided by the twisted mass (horizontal line). Right plot: MC history and distribution $x_{0}=T / 4$ (first strip) together with the euclidean time dependence of the PCAC mass (second strip).

volume and with suitable boundary conditions also for massless Wilson fermions [12]. This is somehow related to the fact that in the region where chiral symmetry is restored only $\mathrm{O}\left(\operatorname{am}_{\mathrm{PCAC}}\right)$ cutoff effects are expected, i.e. very small. On the other side if the mass is of of order $a^{2}$ in general the the $\mathrm{O}\left(a^{2}\right)$ cutoff effects could become the dominant ones.

\subsection{Low energy constants}

The values of the low energy constants (LECs) can be extracted by comparing the results of the numerical simulations for the euclidean time dependence of basic two-point functions, with the prediction of $\chi \mathrm{PT}[13,14]$. In this proceeding we consider the correlation function

$$
C_{\mathrm{P}}\left(x_{0}\right)=\frac{a^{3}}{L^{3}} \sum_{\mathbf{x}} C_{\mathrm{P}}\left(\mathbf{x}, x_{0}\right) \quad \delta^{a b} C_{\mathrm{P}}\left(\mathbf{x}, x_{0}\right)=\left\langle P^{a}\left(\mathbf{x}, x_{0}\right) P^{b}(\mathbf{0}, 0)\right\rangle
$$

between charged pseudoscalar currents

$$
P^{a}(x)=\bar{\chi}(x) i \gamma_{5} \frac{\tau^{a}}{2} \chi(x) .
$$

The euclidean time dependence of the correlation function in $\chi \mathrm{PT}$ is given by

$$
C_{\mathrm{P}}\left(x_{0}\right)=a_{\mathrm{P}}+\frac{T}{L^{3}} b_{\mathrm{P}}\left[\frac{y^{2}}{2}-\frac{1}{24}\right]+\ldots \quad y=\frac{x_{0}}{T}-\frac{1}{2},
$$

where we have defined the following variables

$$
a_{\mathrm{P}}=\frac{B_{0}^{2} F^{4} \rho^{2}}{8} G_{1}(u), \quad b_{\mathrm{P}}=F^{2} B_{0}^{2}\left[1-\frac{1}{8} G_{1}(u)\right] .
$$

Details on the definitions of $\rho, u$ and $g_{1}$ can be found in $[13,14]$. We can thus fit the results from the numerical simulations with the following fit formulæ

$$
C_{\mathrm{P}}\left(x_{0}\right)=A_{0}+A_{2} y^{2} \quad \Rightarrow \quad a_{\mathrm{P}}=A_{0}+\frac{A_{2}}{12} \quad b_{\mathrm{P}}=A_{2} \frac{2 L^{3}}{T} .
$$



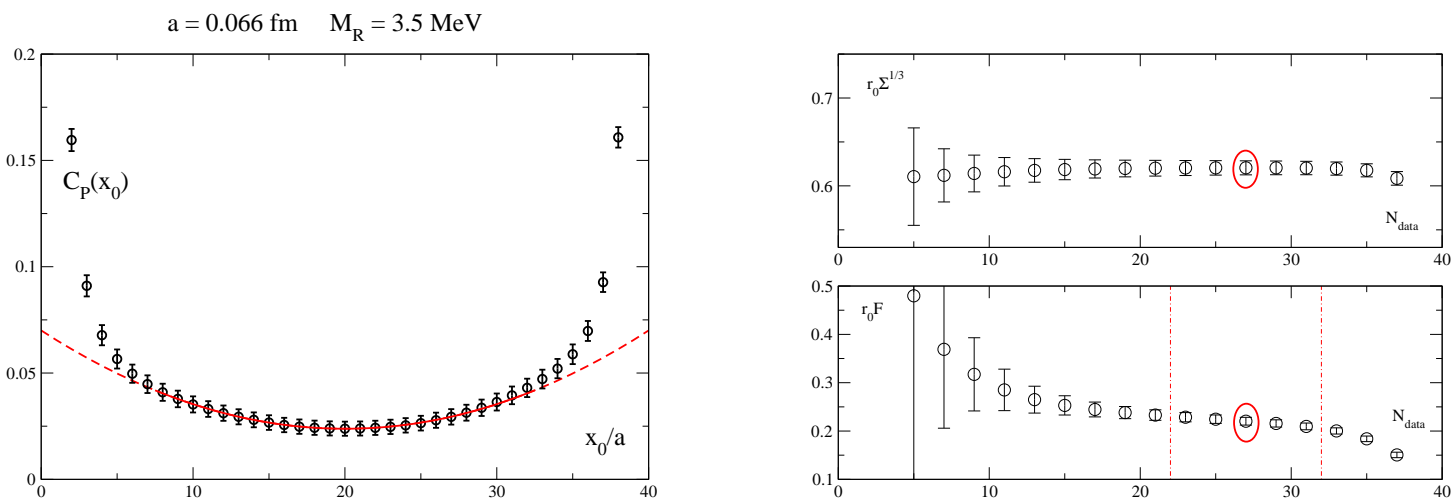

Figure 3: Left plot: Euclidean time dependence of $C_{\mathrm{P}}\left(x_{0}\right)$ together with the result of the fit. The solid line indicates the fit range, while the dashed line indicates the same curve outside the fit range. Right plot: fit results for chiral condensate (first strip) and decay constant (second strip) as a function of the number of points included in the fit around the middle point $T / 2$. The circles indicate the actual values quoted in the text and the dashed lines indicate the range of values used to determine the systematic error.

In the left plot of fig. 3 we show the euclidean time dependence of $C_{\mathrm{P}}\left(x_{0}\right)$ together with the result of the fit (red curve). The line is solid along the fit range and it becomes dashed where the points are not included in the fit. The results of the analysis are

$$
a^{3} L^{3} A_{0}=(5.94(36)) \cdot 10^{-3}, \quad a^{3} L^{3} A_{2}=(4.81(30)) \cdot 10^{-2}
$$

where the errors have been computed with a nested Jackknife/bootstrap procedure. It it is important to check the stability of the fit results against the number of data $N_{\text {data }}$ included in the fit. This is especially important if we want to make sure that the parabolic time dependence is a real feature coming from simulating in the $\varepsilon$ regime and not just accidental, i.e. coming from the standard $\cosh$ dependence which can reproduce a fake parabolic dependence around $T / 2$.

In the right plot of fig. 3 we show the stability of the effective chiral condensate and decay constant as a function of the number of data points (i.e time slices) around the middle point included in the fits. While the chiral condensate shows a remarkable stable result including more point in the fit, the decays constant shows a somehow not completely flat dependence on the number of data points included in the fit. While this is not worrisome, it might be an indication of a physical volume not sufficiently large to suppress higher order corrections. A perfectly well defined way to proceed would is to include in the systematic error for $F$ the spread of its value in the region between the 2 dashed lines. The preliminary result of this analysis is

$$
r_{0} \Sigma^{1 / 3}=0.620(8), \quad r_{0} F=0.220(8)(10)
$$

which compares rather well with results obtained in the $\varepsilon$ regime using improved Wilson fermions [15].

\section{Conclusions and outlooks}

We are establishing the basic knowledge to simulate with Wilson-like fermions in the $\varepsilon$ regime. To do this we have introduced a power counting to study the $\varepsilon$ expansion with Wilson-like fermions. The LO computation for the chiral condensate confirms the absence of any phase transitions, and NLO extension to the condensate and other observables is currently ongoing. 
Numerical simulations in the $\varepsilon$ regime with $\mathrm{Wtm}$ are feasible using a PHMC with exact reweighting. This particular choice allows to lower significantly the quark mass. The extraction of LECs like $\Sigma$ and $F$ then becomes feasible. Moreover the NLO $\varepsilon$ expansion is not contaminated by chiral logs, which could be a benefit to reduce the systematic errors.

Computations in this extreme region with Wilson-like fermions require a detail understanding of the usual systematics: discretization errors, quark mass and volume dependence.

We remark that it might be advantageous to combine $p$ and $\varepsilon$ regime simulations both as a tool to attack $2+1(+1)$ simulations, and to further constrain the values of the LECs.

\section{Acknowledgments}

We thank the organizers of "Lattice 2008" for the very interesting conference realized in Williamsburg.

\section{References}

[1] K. Jansen, A. Nube, A. Shindler, C. Urbach and U. Wenger, Exploring the epsilon regime with twisted mass fermions, PoS LAT2007 (2007) 084 [0711 . 1871].

[2] A. Hasenfratz, R. Hoffmann and S. Schaefer, Reweighting towards the chiral limit, Phys. Rev. D78 (2008) 014515 [0805.2369].

[3] A. Hasenfratz and F. Palombi. Talks presented at this lattice conference.

[4] P. Weisz, Continuum Limit Improved Lattice Action for Pure Yang-Mills Theory. 1, Nucl. Phys. B212 (1983) 1.

[5] Alpha Collaboration, R. Frezzotti, P. A. Grassi, S. Sint and P. Weisz, Lattice QCD with a chirally twisted mass term, JHEP 08 (2001) 058 [hep-lat/0101001].

[6] R. Frezzotti and K. Jansen, A polynomial hybrid Monte Carlo algorithm, Phys. Lett. $\mathbf{B 4 0 2}$ (1997) 328-334 [hep-lat/9702016].

[7] A. Shindler, Twisted mass lattice QCD, Phys. Rept. 461 (2008) 37-110 [0707. 4093 ].

[8] J. Gasser and H. Leutwyler, Thermodynamics of Chiral Symmetry, Phys. Lett. B188 (1987) 477.

[9] A. Shindler, In preparation, .

[10] P. Dimopoulos, R. Frezzotti, V. Lubicz and G. Rossi, ETMC internal notes, .

[11] R. Frezzotti and G. C. Rossi, Chirally improving Wilson fermions. I: O(a) improvement, JHEP $\mathbf{0 8}$ (2004) 007 [hep-lat/0306014].

[12] S. Sint, The Schroedinger functional with chirally rotated boundary conditions, PoS LAT2005 (2006) 235 [hep-lat/0511034].

[13] P. Hasenfratz and H. Leutwyler, Goldstone boson related finite size effects in field theory and critical phenomena with $O(N)$ symmetry, Nucl. Phys. B343 (1990) 241-284.

[14] F. C. Hansen, Finite size effects in spontaneously broken $S U(N)$ x SU(N) theories, Nucl. Phys. $\mathbf{B 3 4 5}$ (1990) 685-708.

[15] A. Hasenfratz, R. Hoffmann and S. Schaefer, Low energy chiral constants from epsilon-regime simulations with improved Wilson fermions, 0806.4586. 